\title{
Star-spots activity on BH Virginis
}

\author{
F.Y. Xiang ${ }^{1}$, S.F. Deng ${ }^{1}$, and Q.Y. Liu ${ }^{2}$ \\ 1 Physics Department of Xiangtan University, Xiangtan, Hunan, P.R.C. 411105, PR China \\ 2 Yunnan observatory, Chinese Academy of Sciences, Kunming, Yunnan, PR China
}

Received May 2, 1996; accepted May 17, 2000

\begin{abstract}
BH Virginis is a short-period RS CVn system. From our observations and archival sources we have gained an ephemeris by means of the times of minima. The light curves and the photoelectric observations in $B$ and $V$ obtained by Koch (1967) have been analyzed by using the Wilson-Devinney program. The principal results are as follows: (1) The mass ratio obtained from the photoelectric light curves is $q=m_{2} / m_{1}=0.967$, close to the spectroscopic value 0.968 obtained by Zhai et al. (1990). (2) The photospheric temperature of the primary, $T_{1}$, at two epochs (1963-1964 and 1991) was about $5945 \mathrm{~K}-$ 6010 K. (3) Several spotted active regions are revealed on both components in 1963-1964 data sets, while only one small spotted active region appears on the secondary star in 1991 .
\end{abstract}

Key words: stars: eclipsing binaries — stars: star-spots

\section{Introduction}

BH Vir is a short-period eclipsing binary $(P=0.81687$ days, G0V $\left.+\mathrm{G} 2 \mathrm{~V}, m_{V}=10^{\mathrm{m}} 8\right)$ with complicated variations in light curves. Abt (1965) published radial velocity observations from which the mass ratio of the two components was found to be $q=m_{2} / m_{1}=1.02$. Zhai et al. (1990) re-analyzed the radial velocity curves by Abt (1965) and determined $q=0.968 \pm 0.046$. The first photoelectric observations of BH Vir were obtained 1953 by Kitamura et al. (1957). Koch (1967) noted the remarkable intrinsic variations of $\mathrm{BH}$ Vir both outside of and within the eclipses. Hoffmann (1982) concluded that the intrinsic variations arose from both components and suggested that BH Vir should join the short-period RS CVn group. Scaltriti et al. (1985) attributed the activity to the primary star. They used their 1984 observations to obtain a value for $\Delta T_{\text {spot }}$, the temperature difference of

Send offprint requests to: F.Y. Xiang the cool spotted region relative to the photosphere, of $1300-1400 \mathrm{~K}$.

Budding \& Zeilik (1987) analyzed photometric observations made by Sadik (1978) and Scaltriti et al. (1985), and found that the cool spotted regions tend to occur at high latitudes (near $45^{\circ}$ ).

Zeilik et al. (1990) collected completed light curves of BH Vir from 1953 to 1986 . They fitted every light curve by a dark, circular spot model for the active regions on the primary star. Their results were $\Delta T_{\text {spot }}=1100 \mathrm{~K}$, latitudes near $45^{\circ}$, longitudes near $90^{\circ}$ and $270^{\circ}$.

Zhai et al. (1990) analyzed photoelectric observations by Koch (1967, observations done in 1963-64 in $U B V$ bands) and by Hoffmann (1982, observations done in 1977 in the $B$ and $5125 \AA$ bands) with the Wilson-Devinney program by using a model with two circular dark spots. However they did not consider any activity regions on the secondary star.

The variations of the light curve of $\mathrm{BH}$ Vir are rather complicated and certainly prevent precise determinations from photometry analysis. In order to better understand the causes of these distortions, we selected this system in our program and made photoelectric observations.

\section{Observations}

The observations of $\mathrm{BH}$ Vir were carried out on 9 nights in April-May 1991, with the $60 \mathrm{~cm}$ reflecting telescope at Yunnan observatory, the Sinica Academy of Sciences, in Johnson's $B$ and $V$ fitters. BD-1 ${ }^{\circ} 2897$ (F5) was chosen as the comparison star and $\mathrm{BD}-1^{\circ} 2868$ (F8) as the check star. Both stars were constant inside the observational errors on all nights. The intrinsic errors of one magnitude difference (variable - comparison) are $\pm 0.020(B)$ and \pm 0 . $017(V)$. These observations and the reduction procedure are published separately (Xiang \& Liu 1997).

Table 1 presents minimum times for $\mathrm{BH}$ Vir calculated with our observations. Our minimum times were obtained with the method of quadratic fitting. All the times of 23 primary minimum and 22 secondary minimum of 
Table 1. Minimum times of BH Vir (Eqs. (1) and (2))

\begin{tabular}{cccc}
\hline JD(HEL) & $E$ & Min & O-C \\
$2400000+$ & & & \\
\hline 48360.1389 & 12551.5 & II & -0.0036 \\
\pm 01 & & & \\
48362.1816 & 12554.0 & I & -0.0025 \\
\pm 01 & & & \\
48389.1385 & 12587.0 & I & -0.0029 \\
\pm 01 & & & \\
48396.0866 & 12595.5 & II & +0.0019 \\
\pm 01 & & & \\
\hline
\end{tabular}

Table 1 and archival sources (Scaltriti et al. 1985; Arévalo et al. 1987) were fitted with both linear and second order ephemeris. Considering the primary and secondary data both separately and combined, we can discard the second order terms, because their errors become larger than the coefficients. No significant difference was found from analyzing the primary and secondary minimum separately, either, indicating a constant period. The linear ephemeris found was:

$$
\begin{array}{r}
\text { MIN I =2438107.19082 }+0.81687060 E \\
\pm 96 \quad \pm 17 \\
\text { MIN II =2438107.1881 }+0.81687055 E \\
\pm 12 \quad \pm 16 .
\end{array}
$$

This is in agreement with the ephemeris calculated by Koch (1967), who quotes:

$\mathrm{JD}=2438107.19047+0.81687099 E$.

Noting that the period has stayed constant to the precision of 1 part in $10^{7}$, which is marginally confirmed by Eq. (1), which gives the $\mathrm{O}-\mathrm{C}$ quoted in Table 1. To the degree of precision of our determinations, the period of BH Vir shows no obvious changes in thirty years.

\section{Photometric solution}

Photometric solutions of BH Vir were obtained by using the 1992 version of the Wilson-Devinney program (Wilson 1992). The individual observations were combined into 64 normal points in each color; the number of individual observations in each normal point was taken as the weight of each point. The two light curves were used simultaneously in deriving our solutions.

Comparing our observations with previous light curves of BH Vir, we can easily see that only a small decrease in the system brightness occurred around phase 0.65 where the systematic deviations by $0.023 \mathrm{mag}(V)$ and $0.022 \mathrm{mag}$ $(B)$ are slightly greater than the intrinsic errors in 1991 although the light curves of the system in 1991 have the missing phases in the beginning of the secondary minimum. The basic astronomical and physical properties of
BH Vir were summarized by Zeilik et al. (1990). The spectral classification of Abt (1965) is in agreement with that of Koch (1967) as for the secondary component (G2V) but there is a difference of about two sub-types in the case of the primary (G0V vs. F8IV-V). From our observations we can determine that the colour index $(B-V)$ of $\mathrm{BH}$ Vir during primary eclipse is about 0.65 . This value is in good agreement with the finding of Koch (1967) and Scaltriti et al. (1985). Therefore we adopted a temperature for star 2 (star eclipsed at Min II) of $5500 \mathrm{~K}$. The other adopted parameters were: gravity-darkening exponents, $g_{1}, g_{2}=0.32$, the bolometric albedo of two components, $A_{1}, A_{2}=0.5$. The linear limb darkening laws were used and the source of the values given by Al-Naimiy (1978). The reflection effect was computed with the detailed model of Wilson (1990). As the spectroscopic mass ratio was found to be $q=m_{2} / m_{1}=1.02$ by Abt (1965), and $q=0.968$ by Zhai et al. (1990), we assumed a series of $q$ values $(0.6,0.7,0.8,0.9,1.0,1.1,1.2)$ to find the preliminary photometry mass ratio with the grid method. The adjustable parameters employed were: the orbitral inclination (i), the dimensionless potentials of star 1 and star $2,\left(\Omega_{1}\right.$ and $\left.\Omega_{2}\right)$, the mean temperature of star $1\left(T_{1}\right)$, and the monochromatic luminosity of star $1\left(L_{1}\right)$. The relative brightness of the secondary star was calculated by the blackbody radiation model.

Because BH Vir seems to be leaving the Main Sequence (see Zhai et al. 1990), the components are reasonably detached their Roche Lobes. It is very important for us to estimate the critical ages for circularization and synchronization for BH Vir. From Zhai et al. (1990) results the absolute parameters of $\mathrm{BH}$ Vir are $m_{1}=0.854 M_{\odot}$, $m_{2}=0.826 M_{\odot}, R_{1}=1.166 R_{\odot}, R_{2}=1.048 R_{\odot}$, $A=4.37 R_{\odot}$. Using the time scales given by Claret \& Cunha (1997), we have found the critical ages for circularization and synchronization for $\mathrm{BH}$ Vir that $t_{\mathrm{syn} 1}=$ $7.1210^{9} \mathrm{yr}, t_{\mathrm{syn} 2}=1.1610^{10} \mathrm{yr}, t_{\mathrm{cir} 1}=6.1910^{9} \mathrm{yr}$ and $t_{\text {cir2 }}=1.1710^{10} \mathrm{yr}$. According to stellar evolutionary theory, we have estimated that the system is older than $1.4910^{10} \mathrm{yr}$. These results reveal that both components are essentially Main-Sequence stars, and they should be synchronized.

Firstly, a convergence test for the solution was carried out using a normal Roche model without spots. The $\Sigma_{i}\left(w_{i}(\mathrm{O}-\mathrm{C})_{i}\right)^{2}-q$ curve is shown in Fig. 1, where $\Sigma_{i}\left(w_{i}(\mathrm{O}-\mathrm{C})_{i}\right)^{2}$ is the sum of weighted squares of residuals between the theoretical light curve and the observations. A minimum of $\Sigma_{i}\left(w_{i}(\mathrm{O}-\mathrm{C})_{i}\right)^{2}$ is achieved at $q=1.0$. At this point, we adopted a dark, circular spot model for the active regions on the primary star and expanded the adjustable parameters to include $q$. The mass ratio converged to $q=0.967$ in the final solution, which is in very good agreement with the spectroscopic and photometric value determined by Zhai et al. (1990). Figure 2 shows the O-C residuals. 


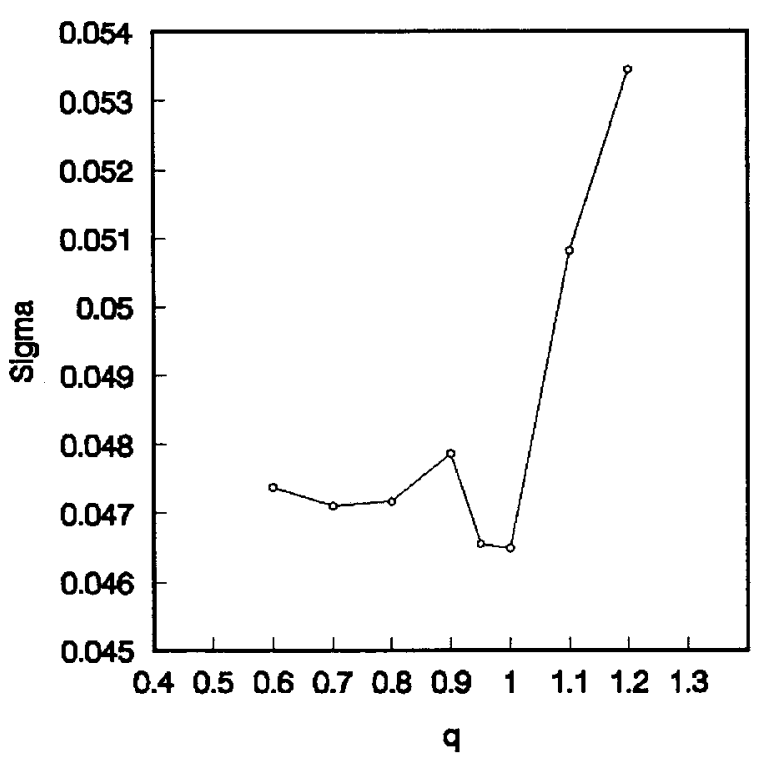

Fig. 1. Variance of the computed fit as a function of the mass ratio $q$

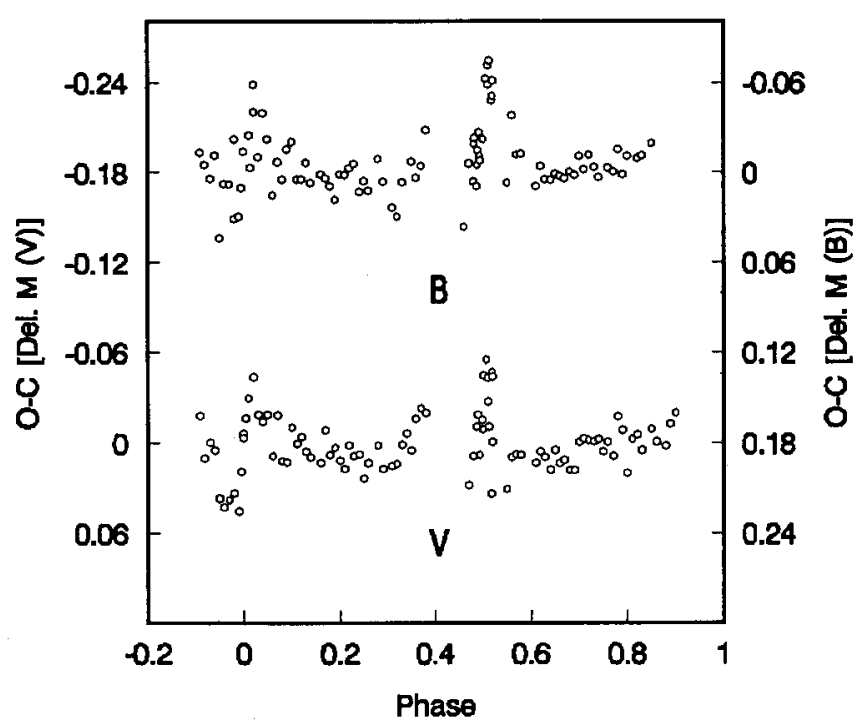

Fig. 2. (O-C) values of BH Vir in 1991 with the model of the spot on the primary star

The theoretical light curves do fit rather satisfactorily the observations, except around the secondary eclipse where the systematic deviations in the $\mathrm{O}-\mathrm{C}$ by $0.020 \mathrm{mag}$ $(V)$ and $0.036 \mathrm{mag}(B)$ are greater than the systematic errors (the computed light curves fainter than observations). This may be caused by some reason of the spot model being wrong, so we suggested that there was no spot on the primary in 1991. Then a cool spot placed on the surface of secondary star was tried (because the secondary star is likely to develop dark-spot activity of the type seen on the Sun). The DC program was used to obtain final spot parameters. The results are given in Tables 2 and 3. The theoretical light curves for the spotted solution are shown

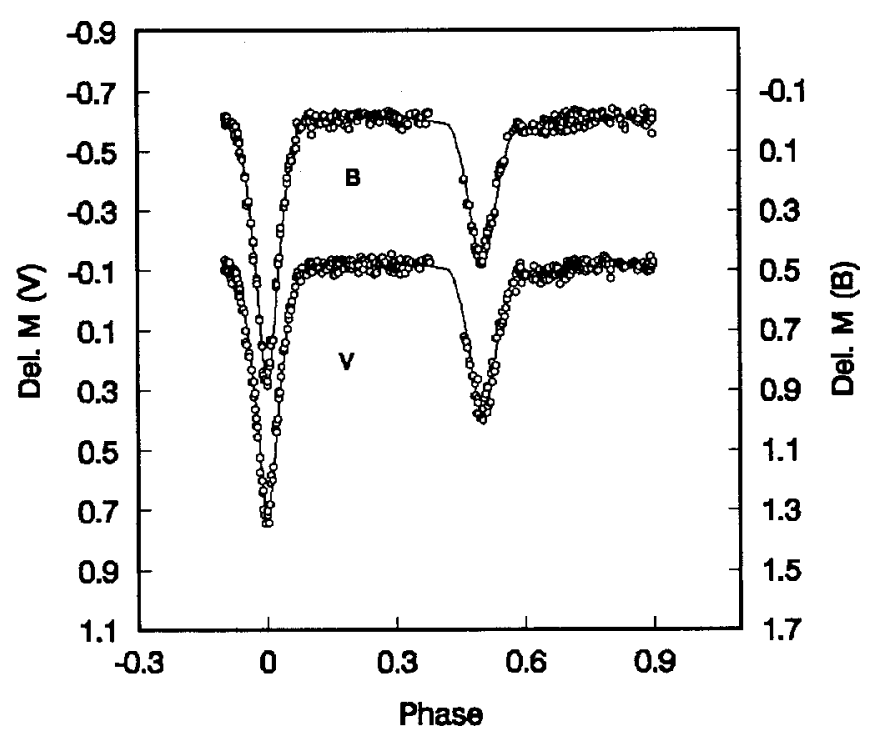

Fig. 3. Light curves of BH Vir in 1991. Open circles represent the observations. Solid lines are theoretical light curves calculated from the parameters in Tables 2 and 3

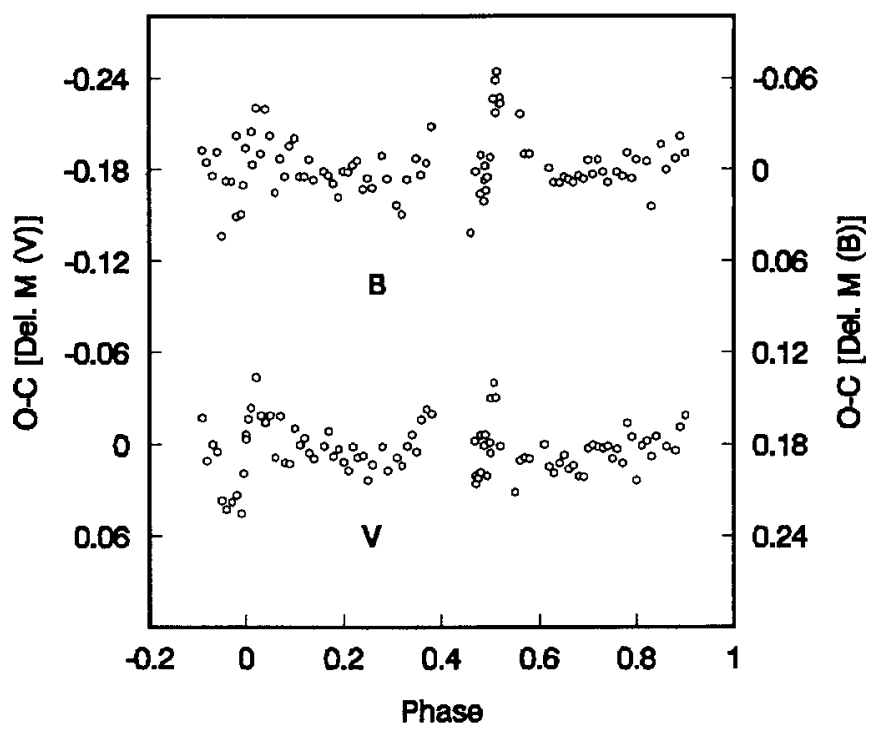

Fig. 4. (O-C) values of BH Vir in 1991 with the model of the spot on the secondary star

as solid lines in Fig. 3. The appropriate $\mathrm{O}-\mathrm{C}$ residuals are shown in Fig. 4. The systematic deviations in the $\mathrm{O}-\mathrm{C}$ residuals are decreased to be $0.003 \mathrm{mag}(V)$ and $0.018 \mathrm{mag}$ $(B)$ which are less than the observations intrinsic errors.

\section{The spot activity in 1963-1964 and 1991}

To study the physical cause of the variations and the distorsions in the light curve of $\mathrm{BH}$ Vir, the archival photoelectric observations in $B V$ by Koch (1967) were also analyzed by us based on the spot model. Zhai et al. (1990) have published an average solution for the observations 
Table 2. BH Vir photometric solution

\begin{tabular}{lll}
\hline Date & $1963 / 64$ & 1991 \\
\hline$L_{1} /\left(L_{1}+L_{2}\right)(V)$ & $0.6716 \pm 0.0054$ & $0.6350 \pm 0.0037$ \\
$L_{1} /\left(L_{1}+L_{2}\right)(B)$ & $0.6905 \pm 0.0053$ & $0.6526 \pm 0.0036$ \\
$X_{1}(V)$ & 0.64 & 0.64 \\
$X_{1}(B)$ & 0.77 & 0.77 \\
$X_{2}(V)$ & 0.66 & 0.66 \\
$X_{2}(B)$ & 0.81 & 0.81 \\
$i$ & $87.43 \pm 0.17$ & $87.44 \pm 0.15$ \\
$\Omega_{1}$ & $4.873 \pm 0.007$ & $4.858 \pm 0.016$ \\
$\Omega_{2}$ & $5.403 \pm 0.005$ & $5.151 \pm 0.024$ \\
$g_{1}$ & 0.32 & 0.32 \\
$g_{2}$ & 0.32 & 0.32 \\
$q$ & $0.967 \pm 0.018$ & $0.967 \pm 0.009$ \\
$A_{1}$ & 0.5 & 0.5 \\
$A_{2}$ & 0.5 & 0.5 \\
$T_{1}(\mathrm{~K})$ & $6010 \pm 5.4$ & $5945 \pm 9$ \\
$T_{2}(\mathrm{~K})$ & 5500 & 5500 \\
$r_{1}$ (pole) & $0.2541 \pm 0.0015$ & $0.2550 \pm 0.0010$ \\
$r_{1}$ (point) & $0.2673 \pm 0.0019$ & $0.2684 \pm 0.0013$ \\
$r_{1}$ (side) & $0.2583 \pm 0.0016$ & $0.2593 \pm 0.0011$ \\
$r_{1}$ (back) & $0.2643 \pm 0.0018$ & $0.2654 \pm 0.0012$ \\
$r_{2}$ (pole) & $0.2193 \pm 0.0016$ & $0.2324 \pm 0.0021$ \\
$r_{2}$ (point) & $0.2265 \pm 0.0019$ & $0.2417 \pm 0.0022$ \\
$r_{2}$ (side) & $0.2217 \pm 0.0017$ & $0.2355 \pm 0.0019$ \\
$r_{2}$ (back) & $0.2251 \pm 0.0018$ & $0.2398 \pm 0.0021$ \\
\hline
\end{tabular}

Table 3. BH Vir starspots parameters

\begin{tabular}{ccccccc}
\hline Data & star & latitude & longitude & ang.radius & $T_{\mathrm{s}} / T_{\mathrm{ph}}$ & $S_{\mathrm{s}} / S_{\mathrm{hem}}$ \\
\hline $1963 / 1964:$ & 1 & 81.0 & 271.30 & 11.50 & 0.71 & $2.5 \%$ \\
& 1 & 76.50 & 91.20 & 11.00 & 0.72 & $2.3 \%$ \\
& 2 & 86.90 & 180.40 & 20.10 & 0.71 & $7.6 \%$ \\
$1991:$ & 2 & 81.40 & 309.50 & 11.20 & 0.650 & $2.4 \%$ \\
\hline
\end{tabular}

Notes: $\mathrm{ph}=$ photosphere hem $=$ hemisphere.

of 1963-1964 and 1977, but they did not consider any activity regions on the secondary star. They gained results of the spotted regions at two epochs being all close to the equator. Their theoretical light curves, around the primary eclipse, could not fit the observations well (see Fig. 5), and the systematic deviations in the $\mathrm{O}-\mathrm{C}$ residuals are greater than $0.050 \mathrm{mag}(V)$ and $0.030 \mathrm{mag}(B)$.

Koch (1967) found that there were night-by-night light curve changes. He suggested that there might be a brighter exterior hemisphere of the cooler component responsible for the depth changes in the primary eclipse. The primary eclipse of the light curve in 1963-1964 is found approximately $0{ }^{\mathrm{m}} 06$ deeper than those in other epochs. We can use the photometric elements given by former authors (Koch 1967; Zhai et al. 1990; Zeilik et al. 1990) to estimate that the primary component is covered by approximately

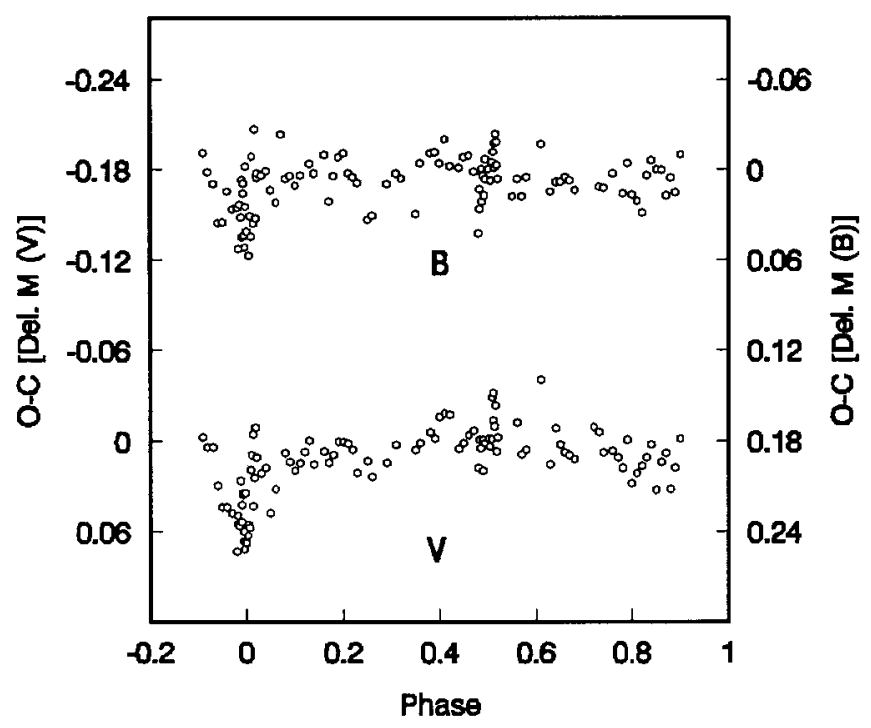

Fig. 5. (O-C) values of BH Vir in 1963/64 for the spot model of Zhai et al.

from $94.8 \%$ to $78.8 \%$ of its hemisphere by the secondary star at the primary eclipse. Hoffmann (1982) and Scaltriti et al. (1985) have emphasized that both components of BH Vir are intrinsically variable and have nonuniform brightness distributions over their surfaces. We considered that the observed distortion, that of the primary eclipse in 1963-1964 being deeper than those in other epochs, could be caused by a cool region on the secondary component, so several groups of cool spots placed on the surface of both components were tried. We used $q, i, T_{1}, \Omega_{1}, \Omega_{2}, L_{1}$ as the adjustable parameters and used Wilson-Devinney program to obtain final solution. The results of the spotted solution are included in Tables 2 and 3 . The fit of the theoretical light curves of our solution to the observations is fairly good and the systematic deviations in $\mathrm{O}-\mathrm{C}$ are less than $0.019 \mathrm{mag}(V)$ and $0.009 \mathrm{mag}(B)$ as show in Figs. 6 and 7 .

From the positions and relative size of the spots on both components for the data of 1963-1964 and 1991, the following conclusions can be drawn:

(1) The spots appear not only on the primary star but also on the secondary star;

(2) The spots on the primary star in 1963-1964 were located in two active longitude belts near $90^{\circ}$ and $270^{\circ}$, but in 1991 they seem to have disappeared;

(3) The photospheric temperature of the primary, $T_{1}$, is inside the range of $5945 \mathrm{~K}-6010 \mathrm{~K}$. This is close to the effective temperature for G0V, the spectral type reported by Abt (1965).

\section{Conclusion}

From 1953 to 1990, a number of authors have made photometric observations of $\mathrm{BH} \mathrm{Vir}$ and have given their 


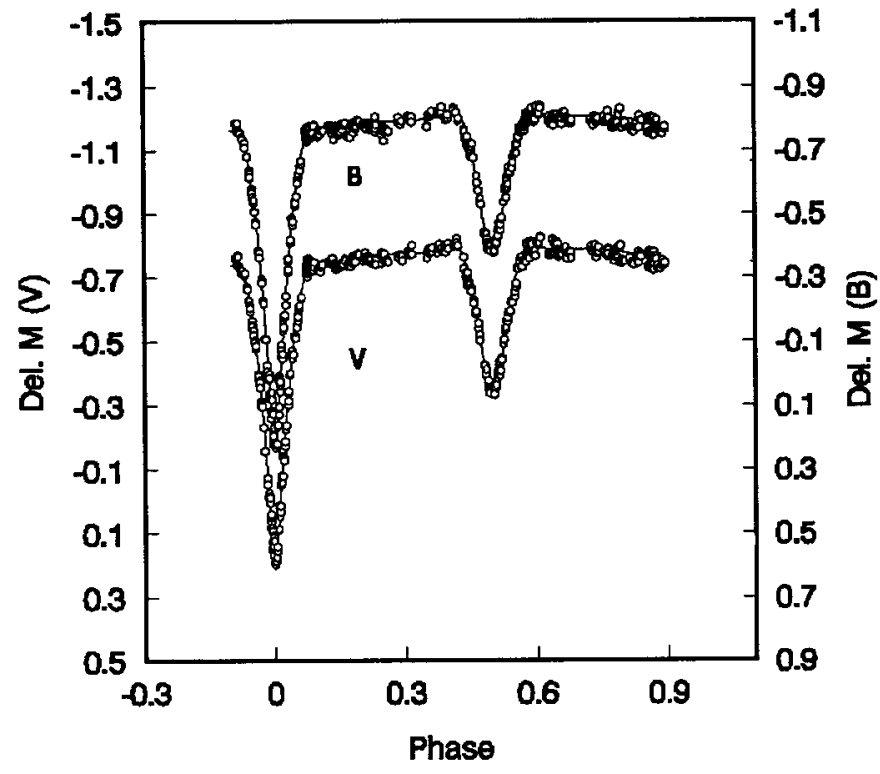

Fig. 6. Light curves of BH Vir in 1963.64. Open circles represent the observations. Solid lines are theoretical light curves calculated from the parameters in Tables 2 and 3

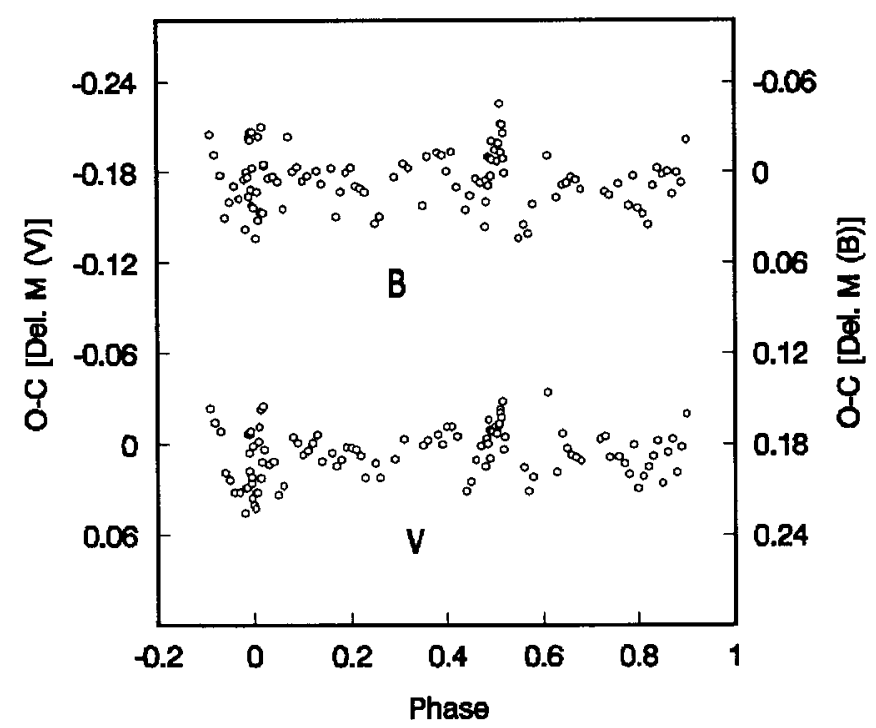

Fig. 7. (O-C) values of BH Vir in 1963/64 calculated from the parameters in Tables 2 and 3

solutions. However, the vast majority of observed light curves show large distortions, so there may be obvious deviations in their results. For instance, the photospheric temperature of the primary, $T_{1}$, has been found different by many authors: about $6100 \mathrm{~K}$ (data from 1963-1964) and $5911 \mathrm{~K}$ (data from 1977, both results by Zhai et al. 1990), $6250 \mathrm{~K}$ (Wilson \& Rafert 1980), and $6000 \mathrm{~K}$ (Zeilik et al. 1990). The light curve of BH Vir in 1991 shows a slight and reasonably smooth curvature excepting only a small decrease around phase 0.65. We have used two different models to fit it, and have obtained $T_{1}=5945 \mathrm{~K}$. We think our solution is reliable.
Table 4. BH Vir spot areas on the primary star

\begin{tabular}{llll}
\hline Data & $S / S_{\mathrm{d}}$ & Wavelength & Source \\
\hline $1963-1964$ & 0.152 & U.B.V & Zhai et al. (1990) \\
$1963-1964$ & 0.067 & B.V & This work \\
1977 & 0.048 & $U . B . V$ & Zhai et al. (1990) \\
1984 & 0.06 & $V$ & Scaltriti et al. (1985) \\
1991 & 0 & B.V & This work \\
& & & \\
$1963-1964$ & 0.020 & B.V & Zeilik et al. (1990) \\
1977 & 0.008 & $V$ & Zeilik et al. (1990) \\
1984 & 0.019 & U.B.V.R & Zeilik et al. (1990) \\
1986 & 0.011 & U.B.V.R.I & Zeilik et al. (1990) \\
\hline
\end{tabular}

Notes: $S_{\mathrm{d}}=$ visible disc.

The most significant result of the present work, in contrast with the results of former authors, is the evidence of the spot active regions not only on the primary star, but on the secondary component, also. In fact, the secondary is a solar-type star, and as such should satisfy the conditions of forming spot active regions. This is further evidence for $\mathrm{BH}$ Vir being a member of the short period RS CVn group.

We note that the average mass ratio $(q=0.967)$ and the radius ratio $\left(r_{2} / r_{1}=0.898\right)$ are less than unity and the visual luminosity of the secondary is about one-half that of the primary (see Table 2). This is in keeping with Zhai et al. photometric and spectroscopic solutions. The greater disparity of luminosities to masses suggests that the primary is approaching the end of its Main-Sequence lifetime, as pointed out by Zeilik et al. (1990).

Table 4 presents the relative size of spots on the primary star. From the changes of the relative areas we can suggest that $\mathrm{BH}$ Vir have active cycle as many authors have already found in other RS CVn systems (Hall 1972; Vogt 1983). However, we must be very cautious about believing these results. First, the observations of BH Vir are not continuous and systematic in epochs. These data can prove that the system is active, but are insufficient to confirm the existence of an activity cycle in BH Vir. Second, all of the former authors did not consider any activity on the secondary. The spot models used by them might be inappropriate, so there may be systematic deviations in their results. Third, the active cycle should be verified by spectroscopic observations. Hence, further observations and detailed study are important to confirm whether $\mathrm{BH}$ Vir have active cycle.

Acknowledgements. We would like to thank Dr. L.P.R. Vaz for his help in calculations and suggestions. The study was supported by Hunan Education Council.

\section{References}

Abt H.A., 1965, PASP 77, 367

Al-Naimiy H.M., 1978, Ap\&SS 53, 181 
Arévalo M.J., Ribayna B., Fuensalida J.J., Bedford D.K., 1987, Vogt S.S., 1983, IAU Coll. 71 "Activity in Red-Dwarf Stars", IBVS 3177

Budding E., Zeilik M., 1987, ApJ 319, 827

Claret A., Cunha N.C.S., 1997, A\&A 318, 187

Hall D.S., 1972, PASP 84, 323

Hoffmann M., 1982, A\&AS 47, 561

Kitamura M., Nakamura T., Takahashi C., 1957, PASJ 9, 191

Koch R.H., 1967, AJ 72, 411

Sadik A.R., 1978, Ph.D. Thesis, University of Manchester (unpublished)

Byrne P.B. and Rodono M. (eds.), p. 173

Wilson R.E., 1990, ApJ 356, 613

Wilson R.E., 1992, Revised Version of Wison-Devinney code (private communication)

Wilson R.E., Rafert J.B., 1980, A\&AS 42, 195

Xiang F.Y., Liu Q.Y., 1997, A\&AS 124, 281

Zeilik M., Leedlow M., Arevalo M., Budding E., 1990, ApJ 354, 352

Zhai D.S., Qiao G.J., Zhang X.Y., 1990, A\&A 237, 148

Scaltriti F., Cellino A., Busso M., 1985, A\&A 149, 11 